\title{
Radiation Risks in a Mission to Mars for a Solar Particle Event Similar to the AD 993/4 Event
}

\author{
Fahad A. Zaman *(D), Lawrence W. Townsend (D) and Naser T. Burahmah (D) \\ Department of Nuclear Engineering, University of Tennessee, Knoxville, TN 37996, USA; \\ ltownsen@utk.edu (L.W.T.); nburahm1@vols.utk.edu (N.T.B.) \\ * Correspondence: fzaman@vols.utk.edu
}

check for updates

Citation: Zaman, F.A.; Townsend,

L.W.; Burahmah, N.T. Radiation Risks in a Mission to Mars for a Solar Particle Event Similar to the AD 993/4 Event. Aerospace 2021, 8, 143. https://doi.org/10.3390/

aerospace 8050143

Academic Editor: Pierre Rochus

Received: 26 April 2021

Accepted: 17 May 2021

Published: 20 May 2021

Publisher's Note: MDPI stays neutral with regard to jurisdictional claims in published maps and institutional affiliations.

\begin{abstract}
Within the past decade, evidence of excess atmospheric ${ }^{14} \mathrm{C}$ production in tree rings, coupled with an increase in annually resolved measurements of ${ }^{10} \mathrm{Be}$ in Arctic and Antarctic ice cores, have indicated that an extremely large solar particle event (SPE) occurred in AD 993/4. The production of cosmogenic nuclei, such as ${ }^{36} \mathrm{Cl}$ in consonance with ${ }^{10} \mathrm{Be}$, indicate that the event had a very energetic "hard" particle spectrum, comparable to the event of February 1956. Herein, we estimate the potential radiation risk to male and female crew members on a mission to Mars that would occur from such an SPE. Critical organ doses and effective doses are calculated and compared with NASA space radiation limits for an SPE comparable to the AD 993/4 event, occurring during the transit phase to Mars, or while the crew members are operating on the surface of Mars. Aluminum shielding, similar in thickness to a surface lander, a spacecraft, and a storm shelter area within the spacecraft, are assumed for the transit phase. For surface operations, including the shielding provided by the atmosphere of Mars, shielding comparable to a spacesuit, enclosed rover, and a surface habitat are assumed. The results of our simulations indicate that such an event might have severe consequences for astronauts in transit to Mars. However, on the surface of Mars, the atmosphere provides some protection against an event similar to the 993/4 SPE. In general, the results show that additional shielding may be required for some of the assumed shielding scenarios.
\end{abstract}

Keywords: space travel; Mars; Martian surface; astronauts; solar particle event

\section{Introduction}

The space radiation environment remains one of the biggest challenges facing future space travel. It consists of three main sources: galactic cosmic rays (GCRs), trapped particles in Earth's magnetic field (TPs), and solar energetic particles (SEPs). Unlike GCRs and TPs which are stable and predictable, SEPs are ejected from the sun through solar particle events (SPEs) in different strengths and in an unpredictable manner. In addition, they can pose a short-term threat to astronauts traveling through space or residing on a lunar or planetary body. Hence, studying the possible impacts of SPEs on future missions could help in risk mitigation planning for such events.

Since SPEs vary unpredictably in their severity, the events that have occurred throughout history provide a database of the possible scenarios that could be used to study the risks of SPEs. SPEs are characterized by their particle fluence level and their energy spectrum. Most events have low proton fluences and pose little or no threat to crews on space missions. However, several times during the approximate 11-year solar cycle, events with large particle fluences, mainly protons, occur. One such event occurred in August 1972. That event had the largest proton fluence of any event in the modern space era. Fortunately, its proton energy spectrum was "soft", meaning that the number of incident particles declined rapidly as the particle energies increased. An event such as this one only poses a radiation exposure threat to crews that are thinly shielded (e.g., by a spacesuit or thin spacecraft shell) [1]. Other events, such as the ones in February 1956 and October 1989, that occurred during the modern space era, had "hard" energy spectra (numbers of incident particles 
declined slowly as the particle energies increased). These events require thicker shields $\left(\sim 20 \mathrm{~g} \mathrm{~cm}^{-2}\right.$ of aluminum) to reduce exposure to below radiation limits [2-4]. Events combining a large proton fluence with a "hard" energy spectrum pose the greatest risk, since they require substantial amounts of shielding to reduce crew exposure to below acute radiation syndrome thresholds and crew radiation limits [5].

Recently, studies of Japanese tree rings discovered rapid increases in ${ }^{14} \mathrm{C}$ content in $\mathrm{AD} 774 / 5$ and $\mathrm{AD} 993 / 4$, which is an implication of a possible extremely large SPE, not of galactic cosmic ray origin. The solar origin of the extreme $774 / 5$ event was confirmed by [6] and the 993/4 event was later confirmed by [7], who studied peaks in production of ${ }^{10} \mathrm{Be}$ and ${ }^{36} \mathrm{Cl}$ in ice cores. Although the AD 774/5 and AD 993/4 events appear to have been single SPEs, a recent study of evidence of ${ }^{14} \mathrm{C}$ increases in Japanese cedar tree rings by [8] suggests that another event which occurred $\sim 2600$ years ago may not have been a single event, but a series of consecutive SPEs over a period of 3 years. Such a series of events could individually yield radiation exposures exceeding short-term limits, and cumulatively yield organ doses and effective doses in excess of career limits. However, unlike the case for the AD 993/4 event and in [9] for the AD 774/5 event, where it is assumed that they were single events, the likelihood of death arising from an acute radiation response may not be significant, unlike the likelihood of that happening for an extremely large, single event. In this work, we examine the possible impact of the recently discovered 993/4 SPE on a trip to the Martian surface. The earlier work by [9] estimated the impact of the 774/5 event on crews on the surface of Mars.

The SPE spectrum is frequently parameterized using a Band function which has event-based spectral fit parameters. When high-energy protons from an SPE interact with Earth's atmosphere, they create secondary neutrons which reach the surface. The Band function utilizes Earth-based neutron monitor data, providing a relatively more reliable high energy fluence estimates. The resulting proton spectra from the Band function are used as source terms to calculate the resulting risk on astronauts using radiation transport/shielding codes.

To estimate the energy spectra of the 993/4 SPE for input into the dose and risk calculations, a scaling factor based on [7,10] was applied to the well-studied February 1956 SPE, which has the hardest energy spectrum measured to date. Several parameterizations were used to model the February 1956 event [11-13]. The recent modified Band function by [13] shall be used in this paper as the baseline spectrum for the applied scaling factor. The scaled spectrum is used to examine the possible radiation effects of an event similar to the 993/4 SPE on future crewed missions to Mars.

In this work, the effects of the 993/4 SPE were studied in two scenarios: a crewed mission on transit to Mars through free space, and a mission on the surface of Mars. To assess the possible risks to the crew, the High $Z$ and Energy TRaNsport (HZETRN) deterministic radiation transport code on the NASA Langley Research Center On-Line Tool for the Assessment of Radiation In Space (OLTARIS) website was used with Computerized Anatomical Male (CAM) and Computerized Anatomical Female (CAF) models placed in a sphere made of an aluminum shielding with varying areal densities representing spacesuits, an enclosed surface rover or lander, and a surface habitat. Finally, the resulting effective and critical organ doses were reported with comparisons to NASA's permissible exposure limits.

Section 2 describes the methodology used in this study with a detailed description of the 993/4 SPE scaled spectrum, the radiation transport code, dose quantities, and the radiation limits for both effective and critical organ doses. Section 3 provides the estimated doses and discusses their implications for future crewed missions. Lastly, Section 4 provides the conclusions of this paper and discusses future work. 


\section{Computational Methods}

The integral energy spectra of the 993/4 SPE is estimated by scaling the February 1956 SPE spectrum. One of the most recent estimates of the February 1956 event proton fluence was provided by [13] through a modified Band function, and is given by

$$
\begin{gathered}
F(>R)=\begin{array}{cc}
J_{1} \times R^{-\gamma_{1}} \times \exp \left(-R / R_{1}\right), & R<R_{b} \\
J_{2} \times R^{-\gamma_{2}} \times \exp \left(-R / R_{2}\right), & R \geq R_{b}
\end{array} \\
R_{b}=\left(\gamma_{2}-\gamma_{1}\right) \times \frac{R_{1} \times R_{2}}{\left(R_{2}-R_{1}\right)} \\
J_{1}=J_{2} \times R_{b}^{\gamma_{1}-\gamma_{2}} \times \exp \left(\gamma_{2}-\gamma_{1}\right)
\end{gathered}
$$

where $F(>R)$ is the integral proton fluence in units of $\# / \mathrm{cm}^{2}$, and $R$ is the proton rigidity in units of GV, while $\gamma_{1}$, and $\gamma_{2}$ are unitless fitting parameters. For the February 1956 event, $R_{1}=0.823 \mathrm{GV}, R_{2}=4.692 \mathrm{GV}, \gamma_{1}=1.822$, and $\gamma_{2}=4.207$, and $J_{2}=1.023 \times 10^{8} \# / \mathrm{cm}^{2}$ [13].

Based on the analysis in [7], which compares the annual mean production of ${ }^{10} \mathrm{Be}$ and ${ }^{36} \mathrm{Cl}$ between February 1956 and other historical SPEs including the AD 993/4 event, the modified Band function was scaled by a factor of $\sim 10$. Figure 1 presents a comparison between the February 1956 SPE spectra and the scaled version representing the AD 993/4 event.

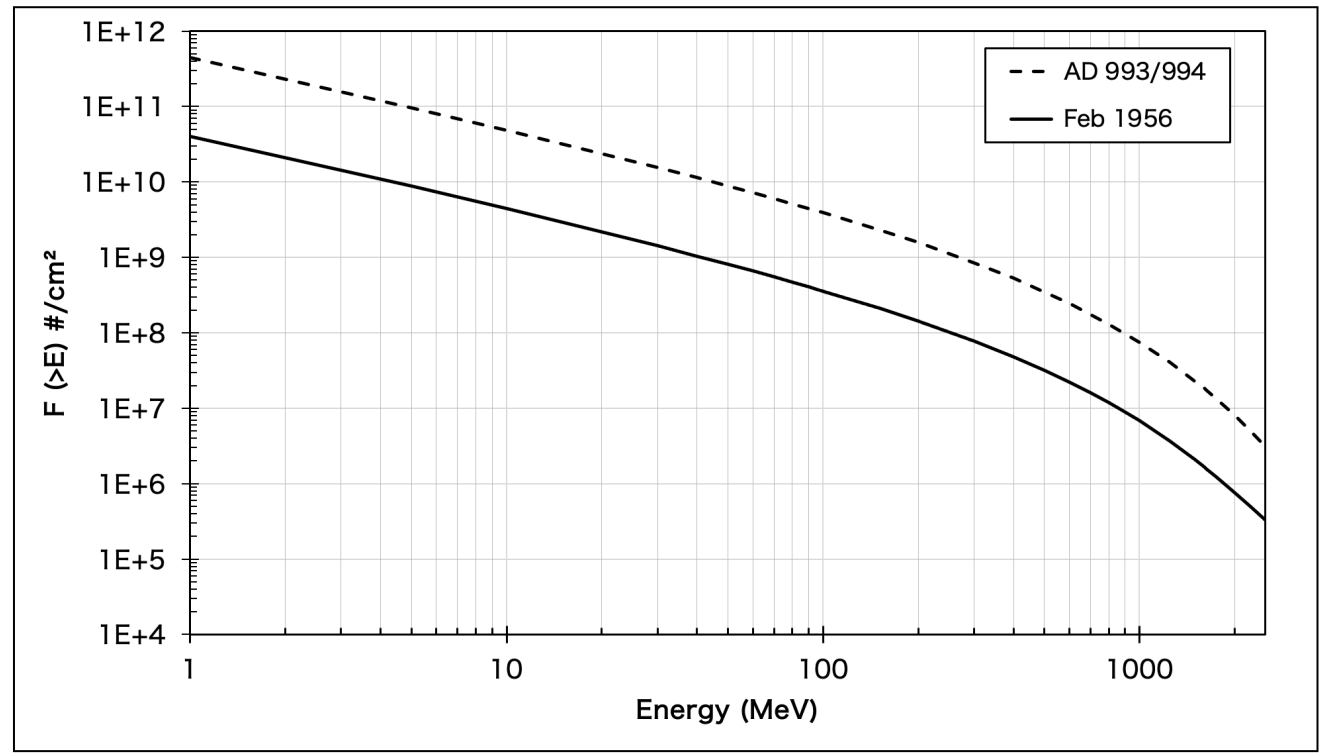

Figure 1. Proton fluence spectra for the February 1956 SPE based on [13] and the scaled AD 993/4 SPE used in this work.

The scaled AD 993/4 spectrum was used as a source term in deterministic radiation transport code to calculate the dose quantities of interest in two scenarios: transit to Mars through free space, and a stay on the Martian surface. The code used in this work is HZETRN, utilized through the OLTARIS website developed by NASA Langley Research Center (https:/ / oltaris.nasa.gov/ accessed on 25-26 March 2021). HZETRN solves the time-independent linear Boltzmann equation using computational methods. Two human phantom models, the CAM and CAF, are used in the geometry to study the effects of SPEs exposure behind aluminum shielding. CAM and CAF models provide details of different organs and their overlying tissues, which enables calculation of the specific dose to each organ, especially since each organ responds differently with different doses of radiation. This helps in determining the severity of the doses from a comparable AD 993/4 event on certain organs. The sensitive organs in this work are lens of the eye, skin, blood forming organs (BFO), heart, and central nervous system (CNS). 
In each scenario, the CAM and CAF models were located separately in the center of a sphere with varying aluminum thickness. For the free space scenario, the thicknesses used are 5, 20, and $40 \mathrm{~g} \mathrm{~cm}^{-2}$. However, since the Martian surface is protected from space radiation by the Martian atmosphere, and since the astronauts are expected to explore the surface in their spacesuits, the thicknesses used are $0.4,5$, and $20 \mathrm{~g} \mathrm{~cm}^{-2}$. Note that an aluminum thickness of $0.4 \mathrm{~g} \mathrm{~cm}^{-2}$ is comparable to a spacesuit, $5 \mathrm{~g} \mathrm{~cm}^{-2}$ to a surface lander or enclosed rover, $20 \mathrm{~g} \mathrm{~cm}^{-2}$ to a nominal transiting spacecraft or surface habitat on Mars, and $40 \mathrm{~g} \mathrm{~cm}^{-2}$ to an SPE "storm shelter" on a spacecraft.

The absorbed dose, $D$, in units of Gray (Gy), is calculated as the absorbed energy in each organ by primary and secondary radiation. However, this physical quantity does not take into account the biological impact of the incident radiation in the organ. Thus, for short-term risk assessment per organ, the absorbed dose is multiplied by the relative biological effectiveness, $R B E$, a unit-less factor that takes into account the biological risk of the incident particle, to calculate the organ dose in units of Gray-Equivalent (Gy-eq). For long-term risk, two unit-less factors are first included to the dose absorbed by the organ: the quality factor, $Q$, that accounts for the biological damage by the incident radiation, and the weighting factor of the organ or tissue $w_{T}$ that takes into account the sensitivity of the organ to the radiation. The doses from all organs are then summed up to constitute the effective dose, $E$, in units of Sievert (Sv). Critical organ doses in units of Gy-eq and effective dose in units of $\mathrm{Sv}$ are provided as outputs from OLTARIS.

The resulting organ dose quantities are then compared to the NASA's permissible dose limits for 30 days, 1 year, and the entire career for the short-term or career non-cancer effects presented in Table 1. They are also compared to the thresholds of severe possible short-term health effects. In addition, the effective dose is compared to NASA's permissible dose limits for 1-year mission for 40-year old astronauts, which is $88 \mathrm{cSv}$ for males and $70 \mathrm{cSv}$ for females [14].

Table 1. NASA's permissible organ dose limits for 30 days, 1 year, and the entire career for short-term or career non-cancer effects [15].

\begin{tabular}{cccc}
\hline Dose & 30-Day & 1-Year & Career \\
\hline Skin (cGy-eq) & 150 & 300 & 600 \\
Heart (cGy-eq) & 25 & 50 & 100 \\
Lens (cGy-eq) & 100 & 200 & 400 \\
BFD (cGy-eq) & 25 & 50 & N/A \\
CNS (cGy) & 50 & 100 & 150 \\
\hline
\end{tabular}

${ }^{*}$ RBE for CNS damage is unknown. Units of cGy are used instead.

\section{Results and Discussion}

Tables 2 and 3 present the organ doses in units of cGy-eq from an event similar to the 993/4 SPE on a mission in a free space transit to Mars for male and female astronauts. At $5 \mathrm{~g} \mathrm{~cm}^{-2}$, for both CAM and CAF, the doses of all organs exceed their uppermost limits for short-term or career non-cancer effects. The eye, skin, heart, and CNS doses at such thickness exceeds the career limit, while the BFO doses exceed the 1-year limit. At $20 \mathrm{~g} \mathrm{~cm}^{-2}$, BFO, heart, CNS doses are still above their corresponding uppermost limits, while eye and skin doses are now below the career limit but above the 1-year limit. This applies to CAM and CAF, which exhibit very similar trends beyond all studied thicknesses when it comes to organ doses. At $40 \mathrm{~g} \mathrm{~cm}^{-2}$, both BFO and heart doses are still above their uppermost limits. However, eye, skin, and CNS doses are now below the 1-year limit, but still above the 30-day limit. 
Table 2. Organ doses from 993/4 SPE for CAM on transit to Mars through free space behind various thicknesses of aluminum shielding.

\begin{tabular}{cccc}
\hline CAM-Free Space & \multicolumn{3}{c}{ Thickness $\mathbf{( g / \mathbf { c m } ^ { 2 } )}$} \\
\hline Does & $\mathbf{5}$ & $\mathbf{2 0}$ & $\mathbf{4 0}$ \\
\hline Eye (cGy-eq) & $8.65 \times 10^{2}$ & $3.15 \times 10^{2}$ & $1.76 \times 10^{2}$ \\
Skin (cGy-eq) & $1.00 \times 10^{3}$ & $3.09 \times 10^{2}$ & $1.71 \times 10^{2}$ \\
BFO (cGy-eq) & $4.58 \times 10^{2}$ & $2.31 \times 10^{2}$ & $1.45 \times 10^{2}$ \\
Heart (cGy-eq) & $3.69 \times 10^{2}$ & $2.13 \times 10^{2}$ & $1.38 \times 10^{2}$ \\
CNS (cGy) & $3.15 \times 10^{2}$ & $1.57 \times 10^{2}$ & $8.99 \times 10^{1}$ \\
\hline
\end{tabular}

Above career limit; 1 Above 1-year limit; 1 Above 30-day limit.

Table 3. Organ doses from 993/4 SPE for CAF on transit to Mars through free space behind various thicknesses of aluminum shielding.

\begin{tabular}{cccc}
\hline CAM-Free Space & \multicolumn{3}{c}{ Thickness $\mathbf{( g / \mathbf { c m } ^ { 2 } )}$} \\
\hline Does & $\mathbf{5}$ & $\mathbf{2 0}$ & $\mathbf{4 0}$ \\
\hline Eye (cGy-eq) & $8.82 \times 10^{2}$ & $3.13 \times 10^{2}$ & $1.78 \times 10^{2}$ \\
Skin (cGy-eq) & $9.97 \times 10^{2}$ & $3.11 \times 10^{2}$ & $1.73 \times 10^{2}$ \\
BFO (cGy-eq) & $4.77 \times 10^{2}$ & $2.37 \times 10^{2}$ & $1.48 \times 10^{2}$ \\
Heart (cGy-eq) & $3.81 \times 10^{2}$ & $2.18 \times 10^{2}$ & $1.40 \times 10^{2}$ \\
CNS (cGy) & $3.34 \times 10^{2}$ & $1.62 \times 10^{2}$ & $9.21 \times 10^{1}$ \\
\hline
\end{tabular}

Above career limit; $\quad$ Above 1-year limit; 1 Above 30-day limit.

The organ doses from an event similar to the 993/4 SPE on the surface of Mars at the mean surface elevation are presented in Tables 4 and 5 . Unlike the free space scenario, behind a thin shield of $0.4 \mathrm{~g} \mathrm{~cm}^{-2}$, which is comparable to a spacesuit, the doses of the eyes, skin, and CNS are below the 30-day limit, while BFO and heart doses are above their corresponding 1-year limits. However, at $20 \mathrm{~g} \mathrm{~cm}^{-2}$, BFO and heart doses are below the 1-year limit, but above the 30-day limit. It is clear from the results that the additional $\sim 20 \mathrm{~g} \mathrm{~cm}^{-2}$ thickness of the atmosphere of Mars provides a fair amount of protection against an event similar to the $993 / 4$ event compared to free space. However, more protection is required to keep some organ doses below NASA's limits.

Table 4. Organ doses from 993/4 SPE for CAM on the surface of Mars behind various thicknesses of aluminum shielding.

\begin{tabular}{cccc}
\hline CAM-Martion Surface & \multicolumn{3}{c}{ Thickness $\mathbf{( g / \mathbf { c m } ^ { 2 } )}$} \\
\hline Does & $\mathbf{5}$ & $\mathbf{2 0}$ & $\mathbf{4 0}$ \\
\hline Eye (cGy-eq) & $8.37 \times 10^{1}$ & $7.47 \times 10^{1}$ & $5.72 \times 10^{1}$ \\
Skin (cGy-eq) & $8.23 \times 10^{1}$ & $7.30 \times 10^{1}$ & $5.56 \times 10^{1}$ \\
BFO (cGy-eq) & $6.72 \times 10^{1}$ & $6.11 \times 10^{1}$ & $4.78 \times 10^{1}$ \\
Heart (cGy-eq) & $6.72 \times 10^{1}$ & $5.79 \times 10^{1}$ & $4.56 \times 10^{1}$ \\
CNS (cGy) & $4.20 \times 10^{1}$ & $3.74 \times 10^{1}$ & $2.72 \times 10^{1}$ \\
\hline
\end{tabular}

Above career limit; $\square$ Above 1-year limit; $\quad$ Above 30-day limit. 
Table 5. Organ doses from 993/4 SPE for CAF on the surface of Mars behind various thicknesses of aluminum shielding.

\begin{tabular}{cccc}
\hline CAM-Martion Surface & \multicolumn{3}{c}{ Thickness $\mathbf{( g / \mathbf { c m } ^ { 2 } )}$} \\
\hline Does & $\mathbf{0 . 4}$ & $\mathbf{5}$ & $\mathbf{2 0}$ \\
\hline Eye (cGy-eq) & $8.47 \times 10^{1}$ & $7.56 \times 10^{1}$ & $5.78 \times 10^{1}$ \\
Skin (cGy-eq) & $8.28 \times 10^{1}$ & $7.35 \times 10^{1}$ & $5.60 \times 10^{1}$ \\
BFO (cGy-eq) & $6.86 \times 10^{1}$ & $6.24 \times 10^{1}$ & $4.87 \times 10^{1}$ \\
Heart (cGy-eq) & $6.45 \times 10^{1}$ & $5.90 \times 10^{1}$ & $4.63 \times 10^{1}$ \\
CNS (cGy) & $4.31 \times 10^{1}$ & $3.83 \times 10^{1}$ & $2.78 \times 10^{1}$ \\
\hline
\end{tabular}

Above career limit; $\square$ Above 1-year limit; $\square$ Above 30-day limit.

To assess the actual biological risk from such doses, we compare the doses of the skin and $\mathrm{BFO}$, which are very radiosensitive, to the thresholds of severe possible shortterm health effects. For the skin, the threshold is $200 \mathrm{cGy}$ for an early transient erythema, $600 \mathrm{cGy}$ for main erythema, and $700 \mathrm{cGy}$ for permanent epilation [16]. It can be seen from Figures 2 and 3 that main erythema is a major concern behind thin shields in free space. Even at $20 \mathrm{~g} \mathrm{~cm}^{-2}$, the skin doses for both CAM and CAF are very close to the early transient erythema threshold. As the shielding thickness approaches $40 \mathrm{~g} \mathrm{~cm}^{-2}$, the skin's doses start to drop below dangerous thresholds in free space, although they are still above the 30-day limit, as seen in Tables 2 and 3. When it comes to the surface of Mars, it is clear again the Martian atmosphere provides protection against severe biological effects to the skin. This is demonstrated in Figures 2 and 3, as all skin dose value for CAM and CAF are below the severe thresholds. On the other hand, for BFOs, the dose at which the surviving fraction is $37 \%$ is $60-160 \mathrm{cGy}$. This is primarily due to the high radiosensitivity of the human stem cells. Figures 4 and 5 illustrate the BFO doses for CAM and CAF. In free space, at $5 \mathrm{~g} \mathrm{~cm}^{-2}$, the BFO dose is above the $37 \%$ surviving fraction threshold, while at 20 and $40 \mathrm{~g} \mathrm{~cm}^{-2}$, is it within the threshold. Thus, in transit to Mars, the astronauts will be at a very high risk from an event similar to the one that occurred in AD 993/4 based on the resulting BFO organ doses. On the Martian surface, the BFO doses are below the 37\% surviving fraction threshold for all the studied thicknesses for CAM and CAF. However, at $0.4 \mathrm{~g} \mathrm{~cm}^{-2}$, the BFO doses are close to the threshold, and thus the astronauts exploring the Martian surface in their spacesuits might be at risk if an event similar to the $993 / 4 \mathrm{SPE}$ reaches Mars.

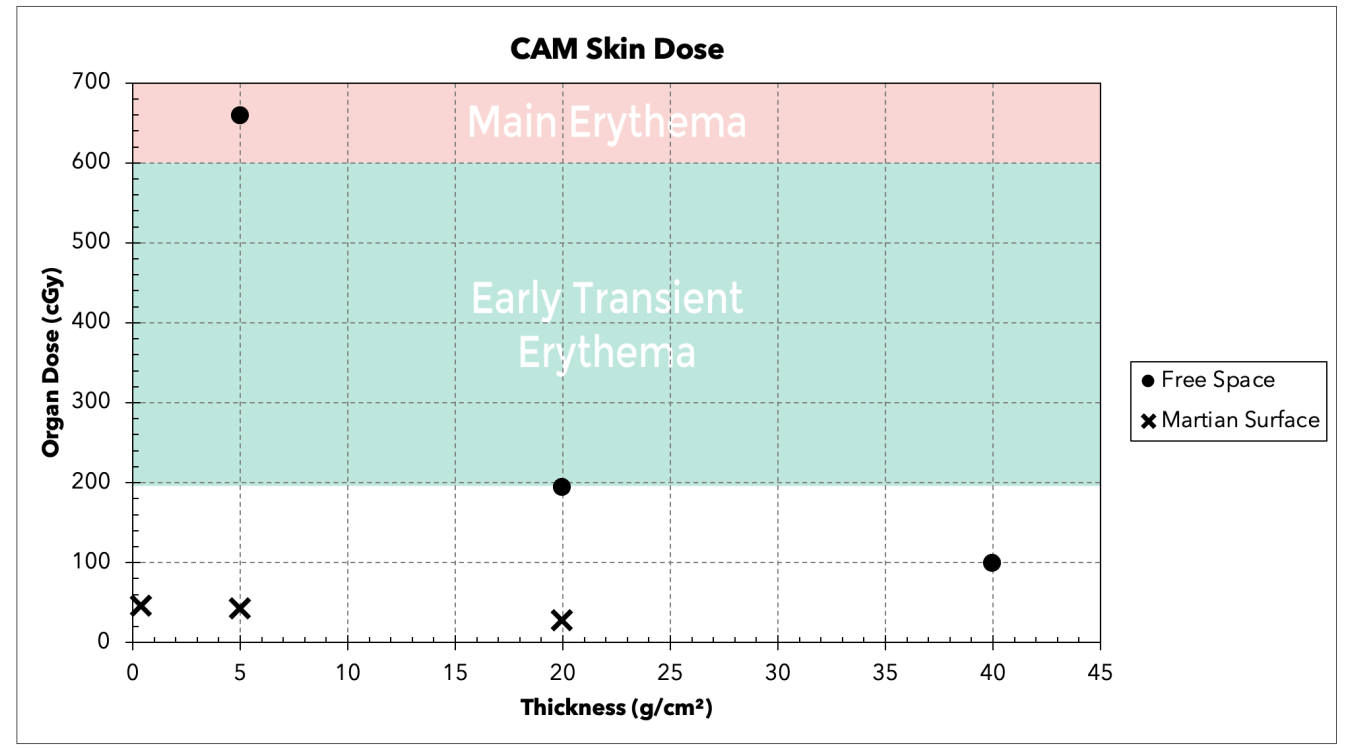

Figure 2. Skin doses from 993/4 SPE for CAM behind various thicknesses of aluminum shielding. The possible biological damage threshold regions are labeled in the background. 


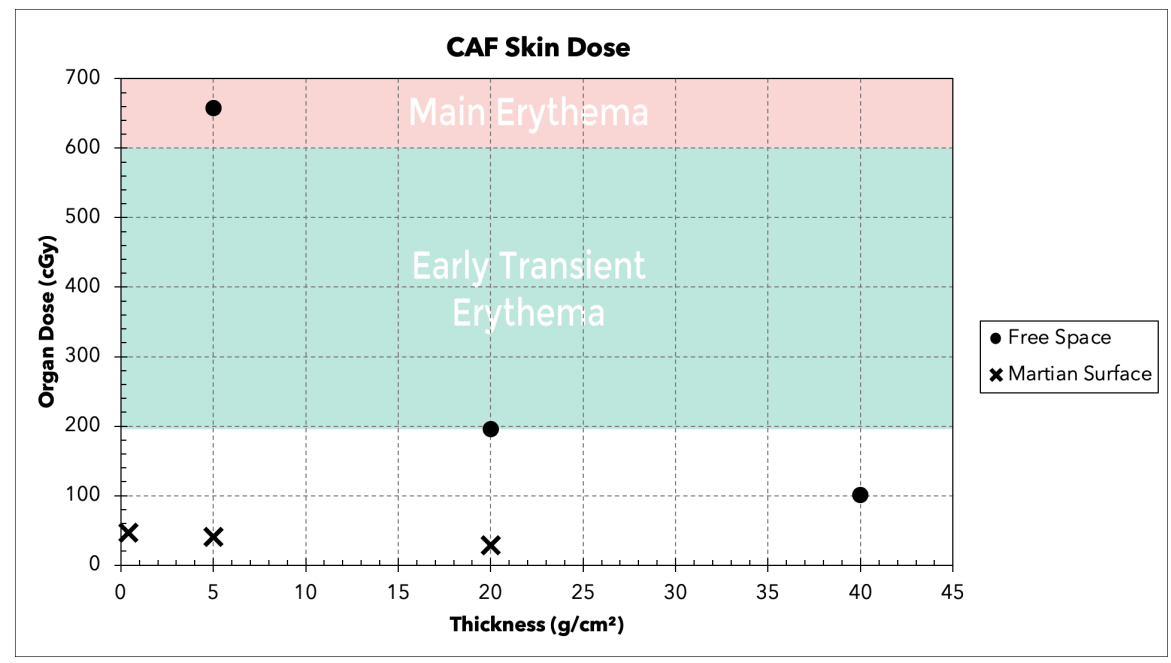

Figure 3. Skin doses from 993/4 SPE for CAF behind various thicknesses of aluminum shielding. The possible biological damage threshold regions are labeled in the background.

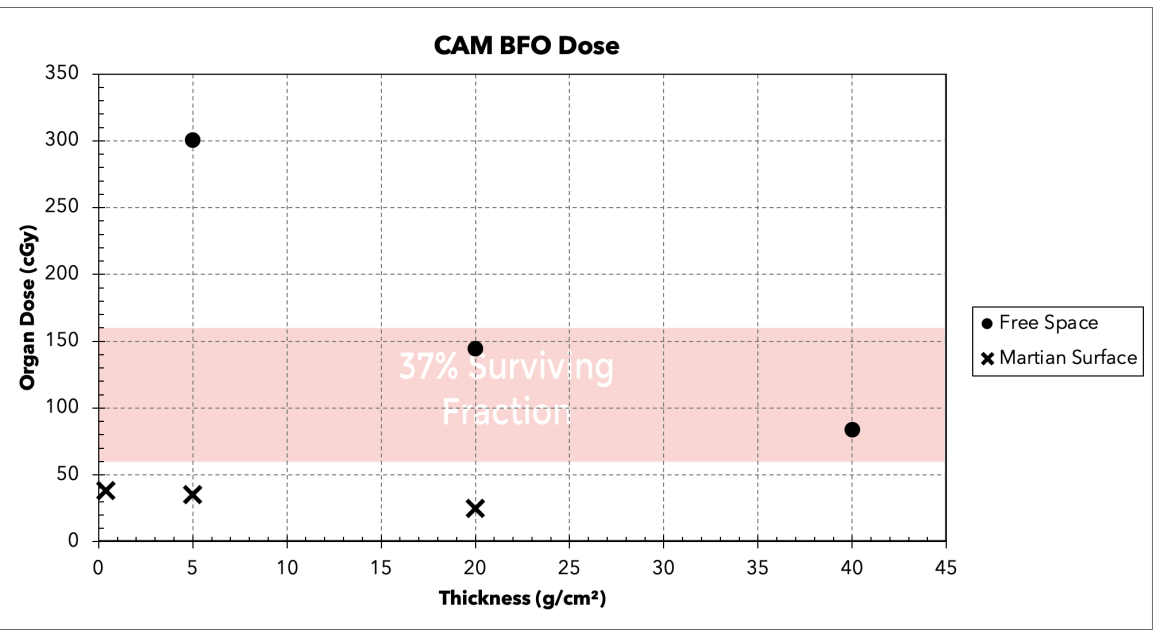

Figure 4. BFO doses from 993/4 SPE for CAM behind various thicknesses of aluminum shielding. The possible biological damage threshold region is labeled in the background.

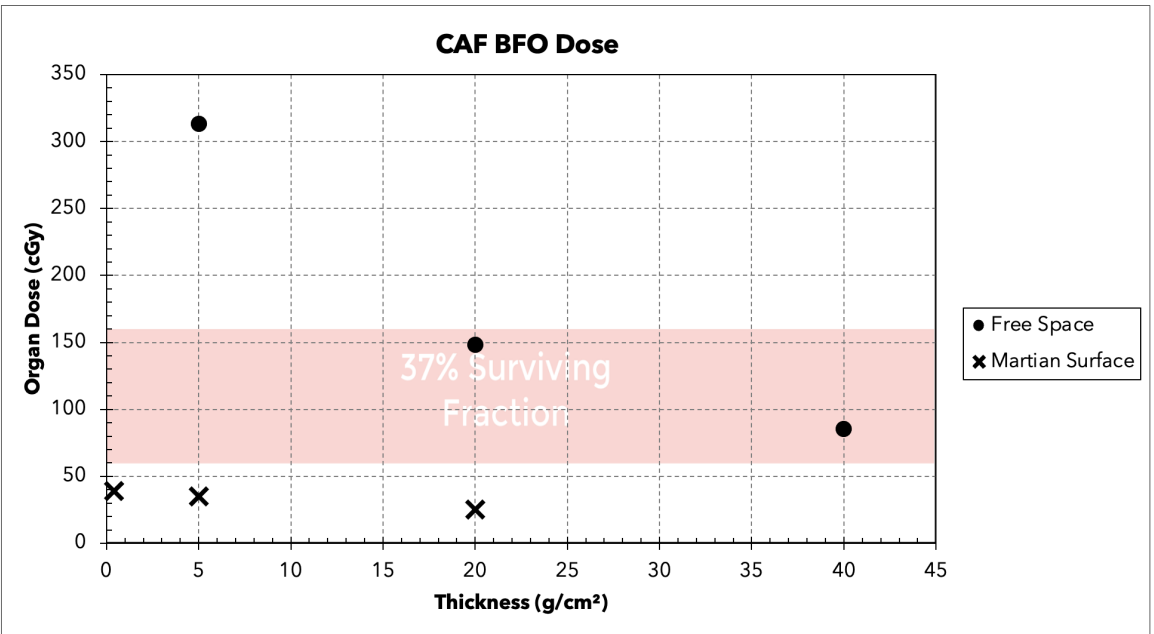

Figure 5. BFO doses from 993/4 SPE for CAF behind various thicknesses of aluminum shielding. The possible biological damage threshold region is labeled in the background. 
Finally, the effective doses for male and female astronauts from an event similar to the 993/4 SPE are presented in Tables 6 and 7. In free space, the effective dose values behind all thicknesses exceed NASA's permissible effective dose limits for 1-year mission for both CAM and CAF. On the other hand, the effective dose values would be below the limits on the Martian surface only behind a thickness of $20 \mathrm{~g} \mathrm{~cm}^{-2}$ or above for CAF. For CAM, a thickness of $0.4 \mathrm{~g} \mathrm{~cm}^{-2}$ is sufficient. Recall that the dose limits vary between male and female astronauts due to biological differences and sensitivities to radiation. A slight increase in CAM's effective dose is observed at $5 \mathrm{~g} \mathrm{~cm}^{-2}$, followed by a decrease with increased shielding. This is probably due to the nuclear fragmentation caused by the secondary particles created by the Martian atmosphere.

Table 6. Effective doses from 993/4 SPE for CAM and CAF on transit to Mars through free space. NASA's permissible effective dose limits for 1-year mission is $88 \mathrm{cSv}$ for 40 -year old males and $70 \mathrm{cSv}$ for 40-year old females [14].

\begin{tabular}{cccc}
\hline Effective Dose-Free Space & \multicolumn{2}{c}{ Thickness $\mathbf{( g / \mathbf { c m } ^ { 2 } )}$} \\
\hline Computerized Model & $\mathbf{5}$ & $\mathbf{2 0}$ & $\mathbf{4 0}$ \\
\hline CAM (cSv) & $5.02 \times 10^{2}$ & $2.66 \times 10^{2}$ & $1.88 \times 10^{2}$ \\
CAF (cSv) & $4.83 \times 10^{2}$ & $2.66 \times 10^{2}$ & $1.88 \times 10^{2}$ \\
\hline Above NASA's permissible limit for 1-year mission
\end{tabular}

Above NASA's permissible limit for 1-year mission.

Table 7. Effective doses from 993/4 SPE for CAM and CAF on the surface of Mars behind various thicknesses of aluminum shielding. NASA's permissible effective dose limits for 1-year mission and $88 \mathrm{cSv}$ for 40-year old males and $70 \mathrm{cSv}$ for 40-year old females [14].

\begin{tabular}{cccc}
\hline Effective Dose-Martian Surface & \multicolumn{3}{c}{ Thickness $\left(\mathbf{g} / \mathbf{c m}^{2}\right)$} \\
\hline Computerized Model & $\mathbf{0 . 4}$ & $\mathbf{5}$ & $\mathbf{2 0}$ \\
\hline CAM (cSv) & $7.52 \times 10^{1}$ & $8.06 \times 10^{1}$ & $5.80 \times 10^{1}$ \\
CAF (cSv) & $7.60 \times 10^{1}$ & $7.07 \times 10^{1}$ & $5.85 \times 10^{1}$ \\
\hline
\end{tabular}

Above NASA's permissible limit for 1-year mission.

\section{Conclusions and Future Work}

The radiation risk of an event similar to the AD 993/4 SPE on a mission to the surface of Mars has been studied in this work for male and female astronauts. In free space, such event might have disastrous effects on the crew, even behind an aluminum shielding of $40 \mathrm{~g} \mathrm{~cm}^{-2}$. This is primarily due to the radiosensitivity of the BFOs. In addition, NASA's permissible dose limits for short-term or career non-cancer effects are all exceeded in free space, along with effective dose limits. On the surface of Mars, the Martian atmosphere provides an adequate protection against an event similar to the AD 993/4 SPE compared to free space. However, the 30-day organ dose limits are exceeded for the heart and BFOs even behind an aluminum shielding of $20 \mathrm{~g} \mathrm{~cm}^{-2}$. Additionally, a thin shielding of $0.4 \mathrm{~g} \mathrm{~cm}^{-2}$, which is comparable to spacesuit, should provide some protection to the BFOs against an event similar to the AD 993/4 SPE; however, the dose received is close to the $37 \%$ surviving fraction threshold, and astronauts should be behind some shielding for protective measures. For female astronauts on the Martian surface, an aluminum shielding of $20 \mathrm{~g} \mathrm{~cm}^{-2}$ is required to keep the effective dose below the limits. Future work will study the risks of an event similar to the AD 993/4 SPE combined with the effects of chronic exposure to the galactic cosmic ray background radiation during the transit to Mars and while on the surface of Mars. Additional future plans include: (1) expanding the AD 993/4 SPE analyses to include a range of altitudes on the surface of Mars; (2) re-analyze the AD $774 / 5$ event using the input spectrum from [13], including additional results for cis-lunar space; (3) analyzing the radiation risks for an event similar to the $660 \mathrm{BCE}$ event; and (4) investigating the effects of spectral hardness uncertainties on the calculated results. 
Author Contributions: Conceptualization, F.A.Z. and L.W.T.; methodology, F.A.Z. and L.W.T.; software, L.W.T.; formal analysis, F.A.Z. and N.T.B.; writing-original draft preparation, F.A.Z., L.W.T., and N.T.B.; writing-review and editing, F.A.Z., L.W.T., and N.T.B.; visualization, F.A.Z. All authors have read and agreed to the published version of the manuscript.

Funding: This research received no external funding.

Institutional Review Board Statement: Not applicable.

Informed Consent Statement: Not applicable.

Data Availability Statement: This study does not report any data.

Conflicts of Interest: The authors declare no conflict of interest.

\section{References}

1. Townsend, L.W.; Nealy, J.E.; Wilson, J.W.; Atwell, W. Large Solar Flare Radiation Shielding Requirements for Manned Interplanetary Mission. J. Spacecr. Rocket. 1989, 26, 126-128. [CrossRef] [PubMed]

2. Townsend, L.W.; Shinn, J.L.; Wilson, J.W. Interplanetary Crew Exposure Estimates for the August 1972 and October 1989 Solar Particle Events. Radiat. Res. 1991, 126, 108-110. [CrossRef] [PubMed]

3. Townsend, L.; Adams, J.; Blattnig, S.; Clowdsley, M.; Fry, D.; Jun, I.; McLeod, C.; Minow, J.; Moore, D.; Norbury, J.; et al. Solar Particle Event Storm Shelter Requirements for Missions Beyond Low Earth Orbit. Life Sci. Space Res. 2018, 17, 32-39. [CrossRef] [PubMed]

4. Zaman, F.; Townsend, L. Radiation Risks in Cis-Lunar Space for a Solar Particle Event Similar to the February 1956 Event. Aerospace 2021, 8, 107. [CrossRef]

5. Townsend, L.; Wilson, J.; Shinn, J.; Curtis, S. Human exposure to large solar particle events in space. Adv. Space Res. 1992, 12, 339-348. [CrossRef]

6. Usoskin, I.; Kromer, B.; Ludlow, F.; Beer, J.; Friedrich, M.; Kovaltsov, G.; Solanki, S.; Wacker, L. The AD775 cosmic event revisited: The Sun is to blame. Astron. Astrophys. 2013, 552, L3. [CrossRef]

7. Mekhaldi, F.; Muscheler, R.; Adolphi, F.; Aldahan, A.; Beer, J.; McConnell, J.; Possnert, G.; Sigl, M.; Svensson, A.; Synal, H.; et al. Multiradionuclide evidence for the solar origin of the cosmic-ray events of AD 774/5 and 993/4. Nat. Commun. 2015, 6, 8611. [CrossRef] [PubMed]

8. Sakurai, H.; Tokanai, F.; Miyake, F.; Horiuchi, K.; Masuda, K.; Miyahara, H.; Ohyama, M.; Sakamoto, M.; Mitsutani, T.; Moriya, T. Prolonged production of $14 \mathrm{C}$ during the $\sim 660 \mathrm{BCE}$ solar proton event from Japanese tree rings. Sci. Rep. 2020, 10, 660. [CrossRef] [PubMed]

9. Townsend, L.; Porter, J.; de Wet, W.; Smith, W.; McGirl, N.; Heilbronn, L.; Moussa, H. Extreme solar event of AD775: Potential radiation exposure to crews in deep space. Acta Astronaut. 2016, 123, 116-120. [CrossRef]

10. O'Hare, P.; Mekhaldi, F.; Adolphi, F.; Raisbeck, G.; Aldahan, A.; Anderberg, E.; Beer, J.; Christl, M.; Fahrni, S.; Synal, H.; et al. Multiradionuclide evidence for an extreme solar proton event around 2610 B.P. (around 660 BC). Proc. Natl. Acad. Sci. USA 2019, 116, 5961-5966. [CrossRef] [PubMed]

11. Tylka, A.J.; Dietrich, W.; Atwell, W. Band function representations of solar proton spectra in ground-level events. In Proceedings of the 38th COSPAR Scientific Assembly, Bremen, Germany, 18-25 July 2010; Volume 38, p. 4.

12. Raukunen, O.; Vainio, R.; Tylka, A.; Dietrich, W.; Jiggens, P.; Heynderickx, D.; Dierckxsens, M.; Crosby, N.; Ganse, U.; Siipola, R. Two solar proton fluence models based on ground level enhancement observations. J. Space Weather. Space Clim. 2018, 8, A04. [CrossRef]

13. Usoskin, I.; Koldobskiy, S.; Kovaltsov, G.; Rozanov, E.; Sukhodolov, T.; Mishev, A.; Mironova, I. Revisited reference solar proton event of 23 February 1956: Assessment of the cosmogenic-isotope method sensitivity to extreme solar events. J. Geophys. Res. Space Phys. 2020, 125, e2020JA027921. [CrossRef]

14. Cucinotta, F.; Schimmerling, W.; Wilson, J.; Peterson, L.; Badhwar, G.; Saganti, P.; Dicello, F. Space Radiation Cancer Risks and Uncertainties for Mars Missions. Radiat. Res. 2001, 156, 682-688. [CrossRef]

15. NASA. 1: Crew Health. In NASA Space Flight Human-System Standard Volume 1, Revision A; National Aeronautics and Space Administration: Washington, DC, USA, 2014

16. Brown, K.; Rzucidlo, E. Acute and Chronic Radiation Injury. J. Vasc. Surg. 2011, 53, 15S-21S. [CrossRef] 